\title{
Cognição e linguagem: introduzindo alguns problemas
}

\author{
Cognition and language: introducing some problems
}

\author{
Carla Viana Coscarelli \\ Universidade Federal de Minas Gerais - UFMG
}

\begin{abstract}
\end{abstract}
In this paper we try to present the connectionst approach of comprehension, and some of the problems that this or any other model of comprehension needs to face. Among the problems, we may mention the necessity to consider the discursive and pragmatic context in which language is used, as well as an embodied perspective of mind - intelligence must be seen as something that happens in a mind that belongs to a body that by its turn lives in a social universe. We also mention the classic problem of how the information that comes from different sources and that is received by different mechanisms in the brains is processed and integrated.

\section{Keywords}

Cognition, Language, Comprehension model

\section{Resumo}

Neste artigo procuramos apresentar uma abordagem conexionista da compreensão e alguns dos problemas que esse ou qualquer outro modelo de compreensão precisa explicar. Entre os problemas, mencionamos a necessidade de considerar o contexto discursivo e pragmático no qual a linguagem é usada, bem como uma perspectiva corporificada da mente - a inteligência deve ser vista como algo que acontece numa mente que pertence a um corpo que, por sua vez, participa de um universo social. Mencionamos também o problema 
clássico de como a informação que vem de diferentes fontes, e que é recebida por diferentes mecanismos cognitivos no cérebro, é processada e integrada.

\section{Palavras-chave}

Cognição, Linguagem, Modelo de compreensão 


\begin{abstract}
"Existem algumas questões sobre o cérebro que são tão misteriosas, tão enigmáticas, que muitos cientistas sérios simplesmente fogem delas, como que dizendo 'Esse seria um estudo prematuro' e 'Eu seria um bobo se embarcasse nessa questão'. E mesmo assim essas são as questões que mais nos fascinam.” (RAMACHANDRAN, 1998, p.188)
\end{abstract}

\begin{abstract}
A té a década de 70, a mente era vista como uma caixa preta que tinha status de mistério e, portanto, não poderia ser estudada. Com o avanço da ciência, muito já podemos dizer sobre ela, embora grandes mistérios ainda permaneçam sem explicação.

A partir dos anos 70, a cognição começou a ser vista pelos pesquisadores como um sistema computacional e a metáfora do cérebro como um computador digital foi largamente usada e muito contribuiu para o desenvolvimento dessas teorias.
\end{abstract}

...como o psicólogo cognitivo Ulric Neisser ressaltou, o computador ajudou a racionalizar o estudo da cognição em si demonstrando que era possível estudar a cognição de uma forma explícita e formal (em oposição aos psicólogos behavioristas da era anterior que argumentavam que os processos internos do pensamento não eram objetos de estudo apropriados). (ELMAN, 2004b, p. 3)

Na década de 80, os trabalhos de Dave Rumelhart and Jay McClelland sobre o processamento paralelo distribuido (PDP) e redes neurais tiveram grande impacto nos estudos da cognição, consolidando uma nova abordagem que passou a ser conhecida como conexionismo.

De acordo com Elman, no conexionismo

o processamento é feito por um (normalmente) grande número de elementos (normalmente muito simples). Esses elementos, chamados nós ou unidades, têm uma dinâmica que é parecida com neurônios. Cada nó recebe input (que pode ser excitatório ou inibitório) de 
outros nós, responde àquele input de acordo com uma função de ativação simples, e torna a excitar ou inibir outros nós com os quais está conectado. Detalhes variam de acordo com os modelos, mas a maioria deles segue esse esquema geral. (ELMAN, 2004b, p. 4)

Em vez de usar representações simbólicas, ou seja, aquelas em que um único nó em uma rede semântica representa um conceito, os sistemas conexionistas consistem em padrões de ativação entre diferentes unidades. Um conceito, por exemplo, seria representado como um padrão de ativações em uma rede.

$\mathrm{O}$ conexionismo defende que todo evento mental deixa um traço na memória; que a memória humana é associativa, sendo assim, a ativação de um elemento na memória provoca a ativação de outros elementos conectados a ele; e que a ativação freqüiente ou consecutiva de elementos na memória aumenta a intensidade das ligações associativas entre esses elementos (FASTREZ, 2002).

Antes dos modelos conexionistas, acreditava-se que o processamento ocorria seriadamente seguindo o fluxo da informação, por exemplo, traços perceptuais de um estímulo eram processados primeiro, gerando uma representação que depois ia passando sucessivamente por outros estágios mais altos (superiores) de processamento. Acreditava-se que a percepção auditiva ou visual era um nível de processamento inferior e que, portanto, ocorria antes de outros processamentos de nível superior, como o reconhecimento da palavra, que, por sua vez, era inferior à construção do significado e, conseqüentemente, ocorria antes dela, e assim por diante. Essa forma de processamento seqüencial é conhecida como bottom-up, ou ascendente. Esse tipo de processamento se mostrou inadequado quando os pesquisadores perceberam a influência do contexto no processamento de letras, sons, palavras e outros estímulos. O efeito da palavra na percepção de uma letra, ou da sentença na compreensão de uma palavra, levou os estudiosos a acreditar num modelo de processamento top-down ou descendente, em que níveis mais altos de processamento influenciavam níveis de processamentos inferiores. O efeito do contexto na percepção, ou seja, a influência de níveis "superiores" nos níveis "inferiores", mostrou que o nosso sistema cognitivo era capaz de trabalhar em vários níveis paralelamente e não apenas em um nível de cada vez.

Com base nisso, no início da década de 80, Rumelhart e McClelland criaram o "modelo de ativação interativa" que se diferenciava dos outros por ser um modelo de processamento paralelo e altamente interativo. Isso significa que, 
nesse modelo, os níveis de processamento realizavam suas tarefas simultaneamente e trocavam informações entre si durante esse processamento, em oposição aos modelos seriados em que apenas um nível de cada vez realizava suas operações e enviava para o nível seguinte, que só então iniciava suas atividades.

Os modelos conexionistas têm, como qualquer outro modelo de outras abordagens, suas deficiências, entre as quais podemos apontar o tratamento da inteligência como sendo individual e como algo que não está inserido em um corpo (descorporificada) e que, conseqüientemente não se insere em um universo social.

A maioria dos modelos de processamento focaliza sua atenção no processamento de palavras ou sentenças, e sabemos que o processamento da linguagem em uma situação natural envolve o discurso e tudo que ele implica, inclusive, muitas sentenças, provavelmente. Os modelos não explicam bem como as representações ou estados construídos para as sentenças funcionam no discurso, nem como o contexto é envolvido nesse processamento, embora essa seja uma preocupação de muitos pesquisadores. ${ }^{1}$ Como se pode notar, muitas questões ainda estão sem resposta, o que significa que ainda há muito trabalho a ser feito.

Em se tratando da linguagem, entendemos essas limitações como aspectos que não podemos deixar de considerar. Nenhum ato de linguagem é individual. A linguagem é um fenômeno social e, portanto, precisa ser vista e tratada como tal. Sendo assim, precisamos de um modelo de leitura que considere esses aspectos como sendo relevantes para explicar a compreensão de textos e hipertextos.

Considerar a mente como sendo corporificada ${ }^{2}$ (MATURANA; VARELA, 2001) e socializada, ou seja, como fazendo parte de um corpo que interage socialmente, leva-nos a pensar na compreensão como um fenômeno ainda mais complexo do que supunham os conexionistas. Núñez e Lakoff (2000) nos mostram como "a matemática é um produto das capacidades neurais dos nossos cérebros, da natureza de nossos corpos, da nossa evolução, do nosso ambiente, e da nossa longa história social e cultural" (p. 9). E assim como a matemática, acreditamos que podemos generalizar essas idéias para todo processamento mental no qual se inclui, inevitavelmente, a leitura. Esses aspectos e seus efeitos precisam ser considerados nos estudos relativos à leitura.

Devemos considerar também uma noção cara à Alife (artificial life) que é o emergentismo, ou seja, 
muitos sistemas têm comportamentos - "propriedades emergentes" - que resultam do comportamento coletivo de componentes do sistema e não da ação de algum componente individualmente. Além disso, esses comportamentos são freqüentemente imprevisíveis. (ELMAN, 2004b, p. 20)

No caso da leitura, essa noção se encaixa na dificuldade que temos de prever que sentido(s) o leitor vai produzir para um texto, dado que são muitos os fatores envolvidos nesse processamento, entre os quais podemos citar as inúmeras capacidades (e limitações) mentais individuais do sujeito, seus objetivos, sua corporificação, o funcionamento social daquele sujeito e daquele texto, naquela situação, etc. Além disso, precisamos estar conscientes de que podemos entender a mente como um sistema dinâmico, no sentido de que seu estado muda continuamente, não havendo, de fato, um estado "final". O significado de uma sentença como um todo, por exemplo, não acontece abruptamente no final da sentença. Pelo contrário, ele é construído gradualmente, e de forma que ainda não sabemos bem explicar, por meio de interações múltiplas e simultâneas entre um grande número de processos mentais e fatores de outras naturezas envolvidos nessa atividade.

Essa forma de processamento, em que os diversos componentes operam simultânea e cooperativamente, um enriquecendo o outro, ajuda-nos a compreender mais facilmente por que "o significado do todo é maior do que se pode prever a partir das palavras consideradas individualmente e das suas relações sintáticas" (ELMAN, et al., no prelo, p. 4). Como o próprio Elman propõe, a linguagem e a cognição de forma geral deveriam ser compreendidas como sistemas dinâmicos (ELMAN, 1995, p. 2). Assim, segundo ele, é mais adequado pensar no estado da rede como “ 'resultado' do processamento de uma palavra, e não como representação dessa palavra” (ELMAN, 1995, p. 16). Nesse sistema dinâmico que ele propõe, os objetos da representação mental são vistos como trajetórias em um espaço mental e não como produto estático.

Hoje acreditamos que o processamento lingüístico requer a integração de informações de vários níveis ou domínios: o léxico, a sintaxe, a semântica, o discurso, o conhecimento de mundo, a situação comunicativa, entre outros. Sabemos também que os dados que um texto apresenta para o leitor, apesar de ricos, não são transparentes nem suficientes para que ele construa sentido e que, para isso, o leitor precisa processar e integrar essas informações, com toda a gama complexa de operações que essas duas ações condensam. 
De acordo com Elman et al. (no prelo ${ }^{3}$ ) o modelo de competição (competition model) proposto por Bates e Mac Whinney (1989) foi um dos primeiros modelos a focalizar múltiplas dicas e a competição entre elas, indo contra a visão mais defendida na época que enfatizava o papel da sintaxe, postulava a sintaxe e a semântica como componentes autônomos na gramática e acreditava que o processamento era modular, havendo assim interações mínimas entre os domínios de processamento (como defendido por Frazier, por exemplo). Mais recentemente, outras abordagens e modelos baseados em expectativas e restrições (constraintbased ou expectation-driven model) desafiaram as abordagens que viam a sintaxe e a semântica como processos separados realizados em diferentes momentos do processamento. Essas propostas ${ }^{4}$ enfatizam os aspectos probabilísticos e contextuais do processamento de sentenças e assumem que a compreensão envolve o uso de informações lexicais, semânticas e pragmáticas para determinar uma análise estrutural.

Mesmo defendendo que as interações (geralmente não-lineares) de diferentes fontes de informação desempenham papel muito importante na compreensão, muitos dos experimentos baseados em modelos de computação inspirados em redes neurais, como simulações conexionistas, ainda são feitos com frases soltas e descontextualizadas.

Muitas vezes me parece que o problema que algumas abordagens computacionais enfrentam não está necessariamente na teoria, mas nas limitações tecnológicas que temos para imitar esses modelos. ${ }^{5} \mathrm{O}$ fato de encontrarmos formas de explicar como a mente funciona não significa que temos de reproduzir isso em outros sistemas, como na informática, por exemplo. Estamos propondo modelos e não realidades virtuais. Os conexionistas e outros estudiosos querem reproduzir os modelos e assim invalidam todos os modelos. De qualquer forma, essa é uma maneira que muitos teóricos encontram para testar seus modelos, que tem enriquecido muito as teorias da cognição.

Elman et al. (no prelo) apresentam boas explicações para a construção de modelos computacionais. Esses modelos nos permitem compreender melhor o que acontece no processamento da linguagem.

Esses modelos computacionais possibilitam detalhar e fazer previsões precisas sobre o comportamento humano. Uma lição desses modelos é que a descrição verbal de um modelo geralmente não leva a previsões claras ou confiáveis sobre que comportamento deveria ser esperado. 
Os modelos podem ser conceptualmente simples, mas o comportamento deles não o é. Um segundo papel desempenhado por tais modelos computacionais é que eles estabelecem as bases para uma análise mais formal dos princípios mais profundos que dão suporte a esse processamento. Finalmente, simulações viabilizam a exploração de um número mais amplo de possibilidades do que os que se revelam nos dados empíricos, e podem servir para gerar hipóteses relacionadas ao comportamento humano que ainda não foram estudadas.

Não há um modelo que dê conta de explicar ampla e efetivamente a cognição. Não podemos ver diretamente o que acontece na nossa mente, nos nossos pensamentos, não podemos ver diretamente como a leitura acontece e como compreendemos textos. O que podemos fazer neste momento é compilar e articular pontos, aspectos, conceitos, formulações que nos forem oferecidos por várias teorias, procurando gerar, a partir desse mosaico, uma possível explicação para a leitura de textos e hipertextos.

Reconhecemos a dificuldade de fazer estudos "in the wild" (HUTCHINS, 1995), ou seja, em uma situação natural, permitindo que muitas variáveis (inclusive não-previstas, bem como variáveis desconhecidas) possam interferir no processamento. Acreditamos que pesquisas de várias modalidades devam ser feitas para que possamos, com os resultados de pesquisas de diferentes naturezas, tentar compreender os processos cognitivos envolvidos na leitura.

\section{Ainda sobre modelos seriados e paralelos}

A discussão sobre modelos de leitura, ou seja, tentativas de explicar como a leitura acontece na mente de um leitor, recai geralmente sob duas grandes vertentes: aqueles que defendem um modelo seriado de processamento e outros que defendem o processamento paralelo.

A fisiologia e neuroanatomia do cérebro nos mostram que ele tem sistemas muito especializados para o processamento de estímulos externos, a ponto de podermos prever, em muitos casos, as conseqüências de uma lesão em determinado ponto desse órgão. Inúmeros casos de lesões cerebrais, como os apresentados e discutidos por Damásio(1996), Sacks (1998), Ramachandram(1998), entre outros, revelam essa especialização dos mecanismos de processamento.

Isso é um dado a favor das teorias modularistas, como a defendida por Fodor (1983). Mas não se opõe às abordagens conexionistas, uma vez que elas 
também defendem que há diferentes níveis de processamento. O que parece ser um ponto dissonante entre essas teorias não é a especialização dos sistemas de processamento, mas a forma de comunicação entre os sistemas, ou seja, os modularistas defendem o processamento seriado, seqüencial ao passo que os conexionistas defendem o processamento simultâneo e interativo das informações.

De acordo com as teorias conexionistas, os estímulos recebidos pelos órgãos sensoriais são imediatamente decompostos e distribuídos entre vários "processadores". Cada neurônio está conectado a um enorme número de outros neurônios e essas conexões permitem que o cérebro realize uma grande quantidade de processamentos simultaneamente.

A quantidade de informação processada pelo cérebro é muito grande para que possamos acreditar que todas as operações devam ocorrer uma após a outra, seguindo uma sequiência previamente determinada. Isso poderia provocar morosidade no processamento, além de poder provocar constantes panes no sistema, pois, se uma operação falhasse, as outras também seriam interrompidas, paralisando o processamento. Mas é possível que algumas operações ocorram seriadamente uma vez que as células no cérebro não disparam juntas, todas ao mesmo tempo, como reação a um estímulo. Disparos acontecem em diferentes momentos, levando-nos a acreditar que é possível que algumas operações aconteçam em resposta a outras (ou como conseqüência do trabalho de outras). Apesar da especialização de alguns sistemas, é difícil sustentar a falta de interação entre eles. Não sabemos qual o grau de interação entre esses sistemas, nem quando ou como essa interação é feita, mas acreditamos que, sobretudo no processamento da linguagem, um nível de processamento afeta o outro. ${ }^{6}$ Este é um ponto que ainda precisa ser muito estudado. Não sabemos como os estímulos são recompostos, como ocorre a integração entre as tarefas realizadas por esses sistemas tão especializados, como esses sistemas geram a compreensão, que nos parece tão integrada, tão global, numa rede tão ricamente conectada.

Geralmente procuramos uma resposta para essa questão tentando encontrar o que costumamos chamar de representação. Tendemos a acreditar que há um produto final nos processamentos mentais, mas precisamos nos lembrar de que a mente é um sistema em constante trabalho. $O$ cérebro não pára de processar, as células do cérebro nunca descansam, elas estão sempre em funcionamento e os estímulos que esses neurônios recebem, no entanto, alteram a sua resposta fazendo o que estamos chamando de ativação. ${ }^{7}$ Sendo assim, esse produto final que procuramos não pode existir. O que deve haver são diferentes 
estados de ativação, em que alguns sistemas de processamento e conexões entre células nervosas são mais estimulados e outros são inibidos, gerando quadros provisórios compostos por diferentes padrões de ativação (resposta ou reação) para cada situação ou conjunto de estímulos recebidos e juntas essas ativações são o que chamamos de representação. A compreensão, portanto, seria um conjunto de ativações de muitas partes do cérebro (e, conseqüentemente, inibição de outras) e não apenas a ativação de uma parte específica dele.

Essa discussão levanta muitas outras questões que precisam ser pesquisadas. Entre elas podemos citar aquelas relacionadas com a seleção dos elementos que serão ativados ou inibidos. Que mecanismos fazem essa seleção? Uma hipótese sobre a base neuronal dessa seleção poderia ser que, enquanto alguns elementos de uma representação cognitiva são ativados, outros são inibidos, possivelmente porque eles representam elementos que são de alguma forma incompatíveis com outros mais fortemente ativados (GRADY, 2000). ${ }^{8}$ Outras questões se referem à forma de organização do conhecimento: "o conhecimento é organizado em unidades muito coerentes e fortemente relacionadas, como domínios, frames, etc. - ou mais livremente distribuído em conjuntos que podem ser mais relacionados entre si ou menos, mas cujos elementos estão disponíveis para recrutamento individual?" A seleção "ativa e depois suprime algum material, ou ela pode escolher elementos conceituais que são, de alguma forma, livres?" (GRADY, 2000, p. 342). Essas são questões que ainda precisam ser respondidas.

\section{Breves considerações finais}

Precisamos ter em mente que, do mesmo jeito que a textualidade não pode ser entendida como sendo a simples justaposição de palavras ou frases e, portanto, bastaria saber como palavras ou frases funcionam para saber como um texto funciona, não podemos acreditar que basta saber como os neurônios funcionam para sabermos como a mente funciona. Uma coisa é o texto, outra é a leitura. Uma coisa é o cérebro e outra é a mente. Por outro lado, não podemos ignorar o funcionamento do cérebro e elaborar teorias que em nada se relacionam com ele. Precisamos elaborar teorias da leitura, ou seja, da compreensão de textos, que levem em consideração as pesquisas de outras áreas, para que, juntos, esses conhecimentos possibilitem uma melhor compreensão do que seja e de como funcione a nossa mente. 


\section{Notas}

${ }^{1}$ Ver, por exemplo, o Landscape Model of Reading, proposto por van den Broek et al. (1999).

${ }^{2}$ The embodiment of mind. "The detailed nature of our bodies, our brains, and our everyday functioning in the world structures human concepts and human reason." (NUNEZ; LAKOFF, 2000, p. 5). A corporificação da mente. "A natureza detalhada dos nossos corpos, dos nossos cérebros e nosso funcionamento cotidiano no mundo estrutura os conceitos e o raciocínio humanos."

The brain and body co-evolved so that the brain could make the body function optimally. Most of the brain is devoted to vision, motion, spatial understanding, interpersonal interaction, coordination, emotions, language, and everyday reasoning. Human concepts and human language are not random or arbitrary; they are highly structured and limited, because of the limits and structure of the brain, the body, and the world. (NUNEZ; LAKOFF, 2000, p. 1). "Cérebro e corpo co-evoluem de forma que o cérebro pode fazer o corpo funcionar bem. Grande parte do cérebro é dedicada à visão, ao movimento, à compreensão espacial, à interação interpessoal, à coordenação, às emoções, à linguagem e ao pensamnento cotidiano. Os conceitos humanos e a linguagem humana não são randômicos ou arbitrários, eles são altamente estruturados e limitados, por causa dos limites e estruturas do cérebro, do corpo e do mundo."

${ }^{3}$ ELMAN, J.; HARE, M.; McRAE, K. Cues, constraints, and competition in sentence processing. In: TOMASELLO, M.; SLOBIN, D. (Eds.). Beyond NatureNurture: Essays in Honor of Elizabeth Bates. Mahwah, NJ: Lawrence Erlbaum Associates. (No prelo).

${ }^{4}$ Como a Competition-Integration Model (SPIVEY-KNOWLTON, 1996, apud ELMAN et al. - no prelo).

${ }^{5}$ É interessante notar como é intrigante a pergunta: o que é mais difícil de fazer, programar um computador para jogar xadrez em nível avançado ou programar um computador para ter uma visão tão boa quanto a de uma criança de dois anos de idade? Assim, percebemos como são complexas as operações feitas pelo cérebro e como ainda não somos capazes de imitar artificialmente as operações que ele parece realizar com tanta facilidade. Muitos exemplos envolvendo a linguagem podem ser dados, entre eles os que envolvem metáforas, ambigüidades, cadeias referenciais complexas, entre outros.

${ }^{6}$ Ver Hofstadter (1995), para uma discussão mais detalhada sobre a relação entre percepção e conceptualização. 
${ }^{7}$ Esse mecanismo no qual a ativação de um conjunto de neurônios leva à ativação de outros tem sido chamado de "ativação espansiva" (spreading activation) pelos cientistas cognitivos que lidam com a linguagem.

${ }^{8}$ Aqui, Grady refere-se a elementos dos espaços input, da teoria dos espaços mentais (FAUCONNIER; TURNER, 2001), que devem ser selecionados serem projetados entre esses espaços.

${ }^{9}$ Grady refere-se à projeção seletiva, ou seja, aos elementos de espaços input que são ativados e que serão projetados em outros espaços.

\section{Referências Bibliográficas}

DAMÁSIO, Antônio R. O erro de Descartes. São Paulo: Companhia das Letras, 1996.

ELMAN, J. L. An alternative view of the mental lexicon. Trends in Cognitive Science. USA: Elsevier, 2004a.

ELMAN, J. L. Connectionism, artificial life, and dynamical systems: new approaches for old questions. San Diego: UCSD, 2004b. Disponível em: <http://cloudbreak. ucsd.edu/ triesch/courses/cogs1/readings/ConnectionismALife DynSys.pdf > .

ELMAN, J. L. Language as a dynamical system. In: PORT, Robert F.; van GELDER, T. (Eds.). Mind as Motion: Explorations in the Dynamics of Cognition. Cambridge, MA: MIT Press, 1995. p. 195-223.

ELMAN, J. L.; HARE, M.; McRAE, K. Cues, constraints, and competition in sentence processing. In: TOMASELLO, M.; SLOBIN, D. (Eds.). Beyond NatureNurture: Essays in Honor of Elizabeth Bates. Mahwah, NJ: Lawrence Erlbaum Associates. (No prelo.)

FASTREZ, Pierre. Navigation Hypertextuelle et acquisition de connaissances. 2002. Tese (Doutorado) - Université Catholique de Louvain, Bruxelas.

FAUCONNIER, Gilles; TURNER, Mark. The Way We Think. Cambridge University Press, 2001.

FODOR, J. The modularity of mind. Cambridge, Mass: MIT Press, 1983.

FRAZIER, L. On comprehending sentences: Syntactic parsing strategies. Bloomington, In: Indiana University Linguistics Club, 1979.

FRAZIER, L. Constraint satisfaction as a theory of sentence processing. Journal of Psycholinguistic Research, 24, 1995, p. 434-468. 
GRADY, Joseph. Cognitive mechanisms of conceptual integration. Cognitive Linguistics, v. 11, n. 3/4, p. 335-345, 2000.

HOFSTADTER, Douglas. Fluid concepts and creative analogies. New York, Basic Books, 1995.

HUTCHINS, E. Cognition in the wild. Cambridge, Mass: MIT Press, 1995.

MATURANA, H. R., VARELA, F.J. A árvore do conhecimento - as bases biológicas da compreensão humana. São Paulo: Palas Athena, 2001.

NÚÑEZ, Rafael; LAKOFF, George. Where mathematics come from? New York: Basic Books, 2000.

RAMACHANDRAN, V. S.; BLAKESLEE, S. Phantoms in the brain. New York: HarperCollins, 1998.

SACKS, Oliver. The man who mistook his wife for a hat. New York: Touchstone. 1970/1998.

van den BROEK, Paul; YOUNG, Michael; TZENG, Yuhtsuen; LINDERHOLM, Tracy. Landscape model of reading: inferences and the online construction of a memory representation. In: OOSTENDORP, Herre van; GOLDMAN, Susan R. (Eds.). The construction of mental representations during reading. New Jersey: Lawrence Erlbaum Associates, 1999. p. 71-98. 